\title{
Sirolimus (rapamicina) en pacientes con hemangioendotelioma kaposiforme. Caso clínico
}

\author{
NATALIE RODRÍGUEZ Z. ${ }^{1,2}$, PABLO BENAVIDES J. ${ }^{3,4}$. \\ 1. Unidad de Hemato-Oncología, Hospital de Niños Roberto del Río. Santiago, Chile. \\ 2. Departamento de Pediatría y Cirugía Infantil, Campus Norte. Facultad de Medicina. Universidad de Chile. Santiago, Chile. \\ 3. Unidad de Hemato-Oncología, Hospital Dr. Gustavo Fricke. Viña del Mar, Chile. \\ 4. Departamento de Pediatría, Facultad de Medicina. Universidad de Valparaíso. Valparaíso, Chile.
}

\begin{abstract}
Sirolimus (Rapamycin) in patients with Kaposiform hemangioendothelioma. Clinical case

Introduction: Kaposiform Hemangioendothelioma (KHE) is a rare vascular tumor, which is associated with the severe coagulopathy known as Kasabach-Merritt phenomenon (KMP). This condition has a mortality rate around $20 \%$. Many treatments have been described, but despite the potentially severe complications that these patients may present, so far there is not a single protocol to follow. International experimental models have used Sirolimus (SRL), a macrolide mTOR inhibitor with antiangiogenic effect. However, its use in children against vascular anomalies, such as KHE, is limited. Objective: To report the development of two patients with KHE and KMP treated with SRL in cancer centers, after multiple failures with other therapies. Case reports: Two infants with severe KHE associated to FKM, resistant to standard therapies and who overcame lifethreatening complications after treatment with SRL are presented. After SRL, the two patients showed normal platelet counts and coagulation tests, reduced lesion size and associated pain, no presence of life threatening conditions or side effects. Conclusion: SRL appears to be effective and safe in treating KHE, representing a new available therapeutic option.

(Key words: Kaposiform hemangioendothelioma, Kasabach-Merritt phenomenon, Sirolimus, vascular tumors).

Rev Chil Pediatr 2013; 84 (5): 537-544

\section{RESUMEN}

Introducción: El hemangioendotelioma kaposiforme (HEK) es un tumor vascular poco frecuente, que se asocia a coagulopatía grave, conocida como fenómeno de Kasabach-Merritt (FKM). Puede ser letal en 20\% de los casos. Se han descrito múltiples tratamientos, pero a pesar de las complicaciones potencialmente severas que
\end{abstract}

Recibido el 27 de marzo de 2013, segunda versión 29 de julio de 2013, tercera versión 9 de septiembre de 2013, aceptado para publicación el 28 de septiembre de 2013.

Este trabajo no contó con apoyo financiero externo.

Este trabajo cumple con los requisitos sobre consentimiento /asentimiento informado, comité de ética, financiamiento, estudios animales y sobre la ausencia de conflictos de intereses según corresponda.

Correspondencia a:

Natalie Rodríguez Z.

E-mail: natalie.rodriguez@redsalud.gov.cl 
pueden presentar estos pacientes, no hay hasta ahora un protocolo único. Existen experiencias internacionales, en modelos experimentales, con el uso de Sirolimus (SRL), macrólido, inhibidor de mTOR, con actividad antiangiogénica. Sin embargo, la experiencia de su uso en niños en el tratamiento de anomalías vasculares como el HEK es limitada. Objetivo: Reportar la evolución de dos pacientes con HEK y FKM tratados con SRL en centros oncológicos, después de múltiples fallas con otras terapias. Casos clínicos: Reportamos dos recién nacidos con HEK profundo asociado a FKM, refractarios a terapias habituales y que evolucionaron con complicaciones amenazantes de la vida, en quienes se inicio tratamiento con SRL. El uso de SRL permitió que los dos pacientes mostraran mejoría en su estado clínico, con normalización del recuento de plaquetas y de las pruebas de coagulación, disminución del tamaño de la lesión, del dolor asociado, desaparición del riesgo vital y sin efectos adversos. Conclusión: SRL parece ser efectivo y seguro en el tratamiento de HEK, representando una nueva opción terapéutica, disponible en nuestro medio.

(Palabras clave: Hemangioendotelioma kaposiforme, Kasabach-Merritt, Sirolimus, tumores vasculares).

Rev Chil Pediatr 2013; 84 (5): 537-544

\section{Introducción}

El Hemangioendotelioma Kaposiforme (HEK) es un tumor vascular poco frecuente, que comprende un grupo variado de lesiones, algunas pequeñas y superficiales y otras profundas, infiltrativas y con riesgo vital (figura 1). En la literatura sólo existe un estudio que reporta una prevalencia estimada de 0,91 casos por 100.000 niños en Estados Unidos ${ }^{1}$. Se presenta generalmente en pacientes menores de 2 años como lesión única, nodular, de crecimiento rápido y progresivo. Este tumor se caracteriza por proliferación endotelial que invade piel, tejido adiposo y músculos. Su localización habitual es en extremidades y tronco, pero se han descrito múltiples ubicaciones, incluso en retroperitoneo $^{1-6}$. Las lesiones más agresivas se asocian al fenómeno de Kassabach-Merrit (FKM), alteración hematológica grave con trombocitopenia severa, coagulopatía de consumo y anemia hemolítica microangiopática, con una alta mortalidad ${ }^{1,3,6}$.

La regresión espontánea o con tratamiento es poco frecuente. Si se produce, puede demorar años, presentando en su evolución, graves problemas ortopédicos y dolor crónico. El diagnóstico definitivo se establece por biopsia. Histológicamente la lesión se caracteriza por proliferación de células endoteliales dispuestas en nódulos tumorales irregulares. La resección es el tratamiento definitivo, lo que frecuentemente no es posible por complicaciones hemorrágicas severas ${ }^{7}$. Se han intentado múltiples tratamientos médicos: corticoides, vincristina, interferón- $\gamma$, quimioterapia combinada, embolización, propanolol, escleroterapia y radioterapia, con eficacia variable, no existiendo consenso sobre el tratamiento de elección ${ }^{1-3,6,8-10}$. En los últimos años, Sirolimus (Rapamicina) un inhibidor del mTOR (mammalian target of rapamycin), ha mostrado ser efectivo en el tratamiento de anomalías vasculares, evidenciando una importante actividad antiangiogénica. Sin embargo, existe experiencia limitada de su uso en el HEK. Recientemente, se han publicado casos de pacientes con HEK refractarios y riesgo vital, en quienes se obtuvo respuesta significativa con Sirolimus (SRL) ${ }^{4,7}$.

Nuestro objetivo fue reportar la experiencia en dos casos clínicos con HEK asociado a FKM, con complicaciones severas, refractarios a los tratamientos habituales y en los que se decidió utilizar SRL, siendo los primeros pacientes con HEK en Chile que reciben este tratamiento, ya que en pediatría su uso estaba limitado principalmente a pacientes con trasplante de órganos sólidos (hígado, riñón) ${ }^{11,12}$.

\section{Casos clínicos}

\section{Caso 1}

Lactante de sexo masculino, ingresó al mes de edad, presentando desde el nacimiento aumento de volumen firme, rojo-violáceo en región anterior muslo izquierdo, de crecimiento progresivo, sin lesiones intraabdominales. Eco-doppler y angioresonancia mostraron tumor de $5,5 \times 4 \times 8 \mathrm{~cm}$, compromete celular 


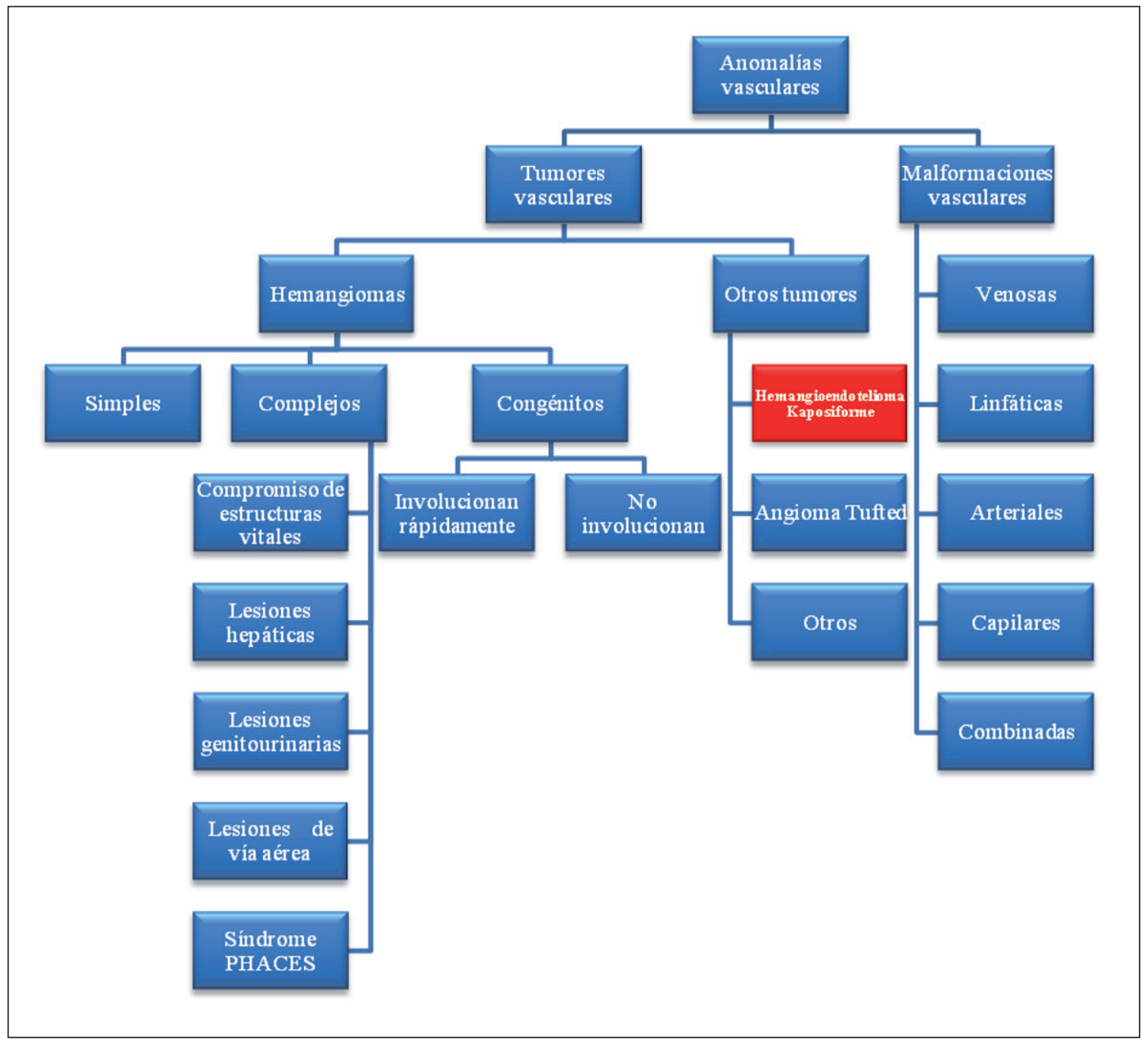

Figura 1. Clasificación de anomalías vasculares en niños (Sociedad Internacional para el estudio de Anomalías Vasculares (ISSVA), 1996).

subcutáneo y plano muscular, sin compromiso óseo, compatible con HEK (figura 2A). Evaluado por equipo multidisciplinario se descartó posibilidad de biopsia y resección quirúrgica, por trombocitopenia y alteración de coagulación severas. Se inició tratamiento con prednisona, ácido tranexámico y transfusión de hemoderivados. Se indicó interferón alfa subcutáneo por aumento de tamaño de la masa (10 x 7,5 cm), presentando un síndrome inflamatorio de respuesta sistémica (SIRS) que requirió conexión a ventilación mecánica por 3 días, no atribuible a cuadro de base, secundario a uso de interferón, por lo que se suspendió. Inició quimioterapia con vincristina y disminución paulatina de corticoides, sin mejoría de alteraciones hematológicas. Al año de tratamiento, la lesión se observaba más pálida, pero de igual tamaño con dolor moderado a severo.

Se decidió intensificar tratamiento utilizando ciclos con vincristina semanal por 3 semanas, actinomicina $\mathrm{D}$, ciclofosfamida y metotrexato semanales cada 21 días por 4 veces, los que recibió sin complicaciones, pero sin respuesta.

A 18 meses del diagnóstico, inició sesiones de escleroterapia percutánea cada 4 a 6 semanas y prednisona. Luego de 12 sesiones, 

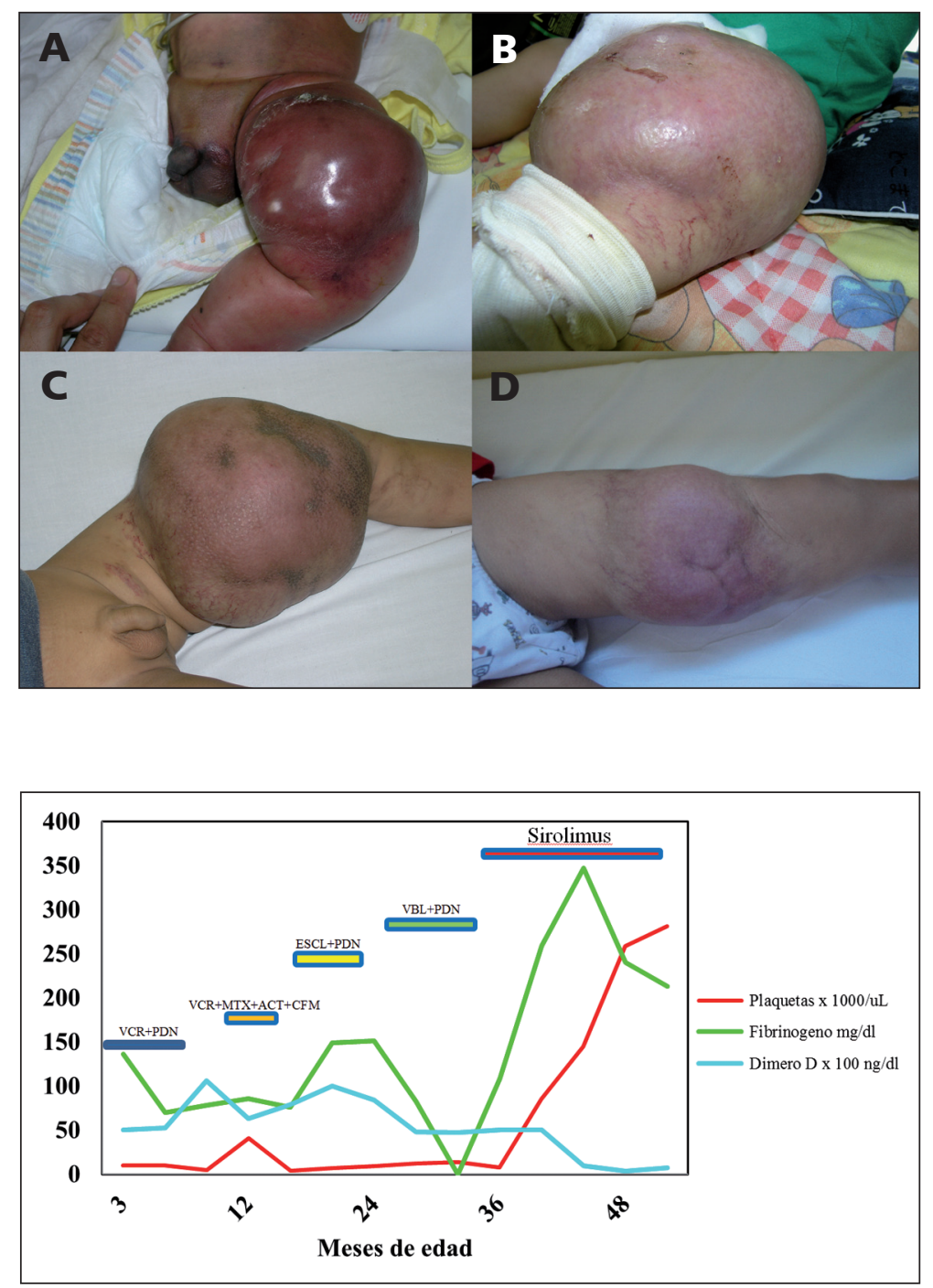

Figura 2. Evolución del HEK caso clínico $n^{\circ}$ 1. A. Al diagnóstico. B. Posterior al tratamiento con escleroterapia. C. Previo al inicio de la terapia con Sirolimus. D. Posterior al uso de Sirolimus por 1 año.
Figura 3. Gráfico de recuentos plaquetas, fibrinogeno y dimero $\mathrm{D}$ según las diferentes terapias del caso clínico $\mathrm{n}^{\mathrm{o}} 1$. $\mathrm{VCR}=$ vincristina, $\mathrm{PDN}=$ prednisona, MTX $=$ metotrexato, ACT $=$ actinomicina, $\mathrm{CFM}=$ ciclofosfamida, $\mathrm{VBL}=$ vinblastina. la lesión se observaba pálida, más blanda, no dolorosa (figura 2B), con mejoría transitoria de exámenes de coagulación (fibrinógeno 145 mg/dL, TTPA normal, TP 84\%), persistiendo anemia moderada, trombopenia severa y dímero D elevado. Evolucionó con reactivación del FKM, lo que contraindica cirugía. Se decidió mantener terapia conservadora con prednisona diaria y vinblastina cada 15 días. Presentó en varias ocasiones crisis de dolor severo requiriendo uso de opiáceos. No se evidenciaron modificaciones en el tamaño tumoral (figura 2C) y presentó empeoramiento de los parámetros hematológicos (plaquetas $7.000 \mathrm{x} \mathrm{mm}^{3}$, fibrinógeno bajo, TP y TTPA prolongados). En estas condiciones sufrió TEC con hemorragia subaracnoidea, tras una caída en su domicilio. Ingreso a UCI, no requirió tratamiento quirúrgico. Recibe transfusión de hemoderivados y ácido tranexámico, además de anticonvulsivante por 4 meses.

A los 3 años y 6 meses de edad, con el consentimiento de los padres, se indicó SRL en dosis de $0,8 \mathrm{mg} / \mathrm{m}^{2} /$ dosis, 2 veces al día, vía oral. A los 3 meses se observó normalización de exámenes hematológicos, desaparición del dolor y paulatina disminución de masa tumoral (figura 3). Mantuvo niveles plasmáticos en 


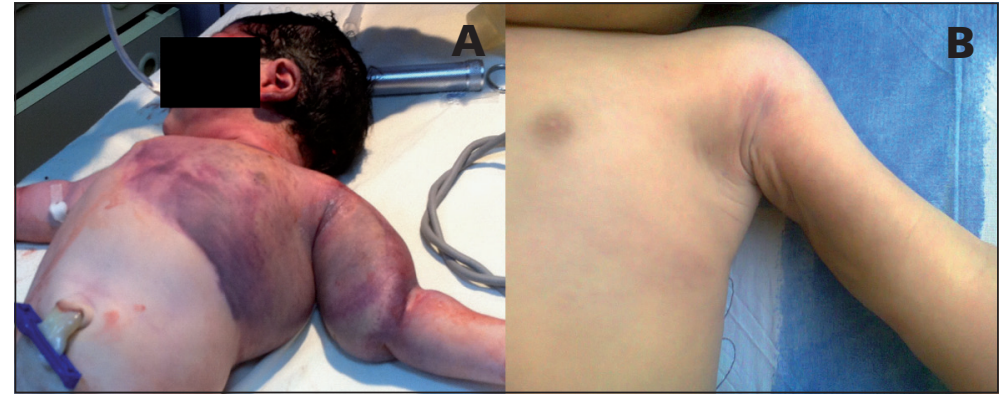

Figura 4. Evolución del HEK caso clínico $n^{\circ} 2$. A. Previo al inicio de tratamiento. B. Después de un año de tratamiento. rango terapéutico. No presentó complicaciones asociadas al uso de SRL, el que se mantendrá hasta completar 2 años (figura 2 C y D).

\section{Caso 2}

Neonato, sexo femenino, nació con asfixia neonatal, requiriendo reanimación cardiopulmonar. Cursó con encefalopatía hipóxico-isquémica, hipertensión pulmonar, insuficiencia renal aguda y shock, que evolucionaron satisfactoriamente. Desde el nacimiento presentó parálisis braquial izquierda y aumento de volumen rojo-violáceo del brazo, hombro y hemitórax izquierdo (figura 4A). Se sospechó una alteración vascular, pero ecografía informó sólo edema de partes blandas. Cursó persistentemente con trombocitopenia y anemia severas, asociado a coagulopatía ( $\mathrm{Hb} 10,5 \mathrm{gr} /$ $\mathrm{dL}$, plaquetas $17.000 \times \mathrm{mm}^{3}$, TP $63 \%$, fibrinógeno $115 \mathrm{mg} / \mathrm{dL}$, dímero $\mathrm{D}>10.000 \mathrm{ng} / \mathrm{mL}$ ), recibiendo múltiples transfusiones de hemoderivados.

A los 12 días presentó dificultad respiratoria y derrame pleural izquierdo hemorrágico. Se reevaluó con ecografía y angiotomografía, que mostraron lesión vascular hemangiomatosa extensa de $13 \times 7 \times 4 \mathrm{~cm}$, del brazo, hombro y hemitórax izquierdo, con derrame pleural (figura 5), compatible con HEK asociado a FKM. Se consideró inoperable y no se realizó biopsia por riesgo de hemorragia.

Inició terapia con prednisona más propanolol con reducción del tamaño tumoral, resolución del derrame pleural y mejoría del FKM. Se completaron 7 días con corticoides y se mantuvo con propanolol. Sin embargo, presentó reactivación del FKM, reiniciando a los 25 días corticoides en dosis altas, sin respuesta, con importante crecimiento tumoral hacia hemitórax contralateral y compromiso critico del paciente. A los 34 días se asoció vincristina, observándose regresión leve del tamaño, pero persistencia del FKM.

Debido a refractariedad al tratamiento habitual y a la presencia de complicaciones graves, con el consentimiento de los padres, a los 55 días se agregó SRL $\left(0,8 \mathrm{mg} / \mathrm{m}^{2} /\right.$ dosis, 2 veces al día). A los 40 días de su inicio, asociado a vincristina y corticoides, se obtuvo remisión del FKM (figura 6) y disminución significativa del tamaño tumoral. Continuó tratamiento ambulatorio, con niveles plasmáticos de SRL en rango terapéutico (5 y $15 \mathrm{ng} / \mathrm{mL})$. Después de 9 meses de tratamiento, la niña presentó remisión clínica, que se ha mantenido al año de edad (figuras 4B y 6), persistiendo paresia braquial. No se observaron efectos adversos atribuibles a SRL.

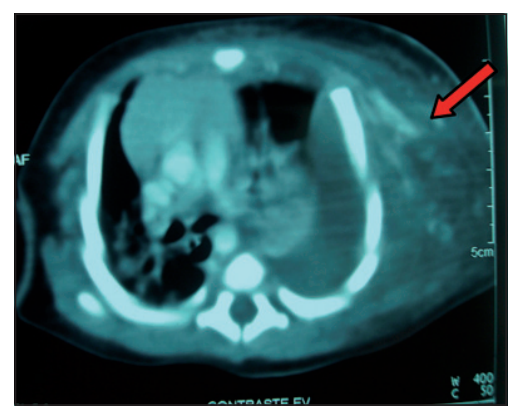

Figura 5. Angiotomografía computada al diagnóstico del caso clínico $\mathrm{n}^{\circ} 2$ que muestra masa vascularizada extensa de $13 \times 7 \times 4 \mathrm{~cm}$, de la región axilar y de la pared del hemitórax izquierdo y derrame pleural del mismo lado. 


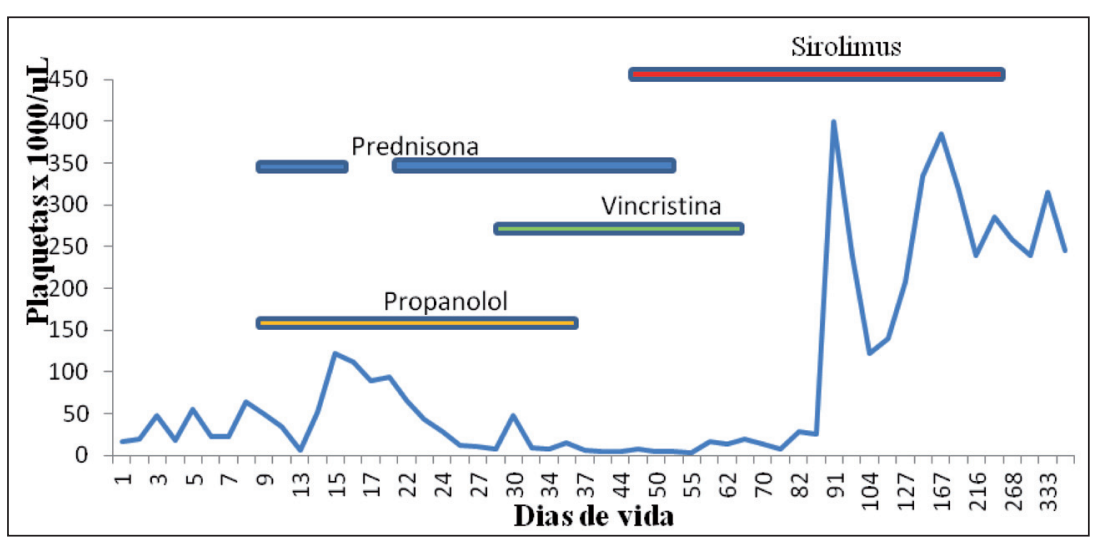

Figura 6. Se grafica la evolución del recuento plaquetario en relación con los diferentes tratamientos desde el nacimiento al año de edad del caso clínico $n^{\circ} 2$.

\section{Discusión}

El HEK, descrito inicialmente como un hemangioma capilar gigante, corresponde a un tumor vascular, diferente en su patrón inmunohistoquímico de otras anomalías vasculares. El riesgo de hemorragia generalmente impide biopsiar y el diagnóstico se basa en los hallazgos clínico-radiológicos, como en nuestros ca$\operatorname{sos}^{1-3,5}$.

Su morbimortalidad está determinada por el efecto compresivo de la masa tumoral y el FKM, complicaciones observadas en nuestros pacientes. La patogénesis del FKM involucra consumo local de plaquetas, favoreciendo el crecimiento de la lesión y desencadenando una coagulopatía, con alta letalidad (14-24\%). En este contexto, la transfusión de plaquetas debería restringirse a episodios de hemorragia con riesgo vital o cirugía. Además el crecimiento tumoral puede causar destrucción de tejidos con fibrosis, anomalías ortopédicas y dolor crónico severo ${ }^{1,8-10,13,14}$.

Se han utilizado múltiples terapias, pero ninguna ha demostrado clara efectividad $^{1-3,6,8-10}$. Los esteroides son la terapia de primera línea, pero son exitosos en un 10\%. La vincristina se administra generalmente asociada a corticoides, ya que la "monoterapia" no sería beneficiosa en pacientes con HEK muy sintomáticos ${ }^{2,6,10,13}$. Con Interferón alfa se logra respuesta en $60 \%$ de los casos, pero casi un tercio de los pacientes presentan complicaciones neurológicas severas y síndrome de distress respiratorio grave, como en el caso 1 . Se han reportado respuestas exitosas con el uso de quimioterapia combinada con ciclofosfamida, metrotexato y actinomicina, sin embrago, faltan estudios randomizados que comparen su eficacia ${ }^{8}$.

El mTOR es una kinasa serina/treonina, regulada por la fosfoinositol-3-kinasa (PI3K) que participa en la angiogénesis. En modelos murinos se demostró que su alteración y la de otros miembros de la vía $\mathrm{PI} 3 \mathrm{~K} / \mathrm{mTOR}$ produce malformaciones vasculares. Se postula entonces que agentes inhibidores de mTOR tendrían un potencial antiangiogénico y jugarían un rol en el tratamiento de anomalías vasculares como el HEK (figura 7). SRL es el único inhibidor de mTOR aprobado por la FDA, con un perfil de seguridad conocido, siendo bien tolerado en niveles plasmáticos entre 5 y 15 $\mathrm{ng} / \mathrm{mL}$, con escasos efectos adversos descritos como hiperlipemia, mucositis, hipertensión arterial y elevación de transaminasas ${ }^{4,7}$. Actúa como inmunosupresor, por lo que se describe aumento del riesgo de desarrollar segundos tumores como linfomas, y también infecciones, por lo que se recomienda profilaxis para Pneumocystis jirovecii ${ }^{4}$.

Nuestros pacientes recibieron las terapias descritas con escasa o nula respuesta, evolucionando con complicaciones graves. El reporte de casos de HEK refractarios con excelente respuesta al uso de SRL, muestra que es una droga segura ${ }^{4,7}$. Estas experiencias y su disponibilidad en nuestro medio (Rapamicina, Rapamune $\AA$, Wyeth, USA, solución oral $1 \mathrm{mg} /$ $\mathrm{mL}$ ), nos motivaron a utilizarlo. Los pacientes 


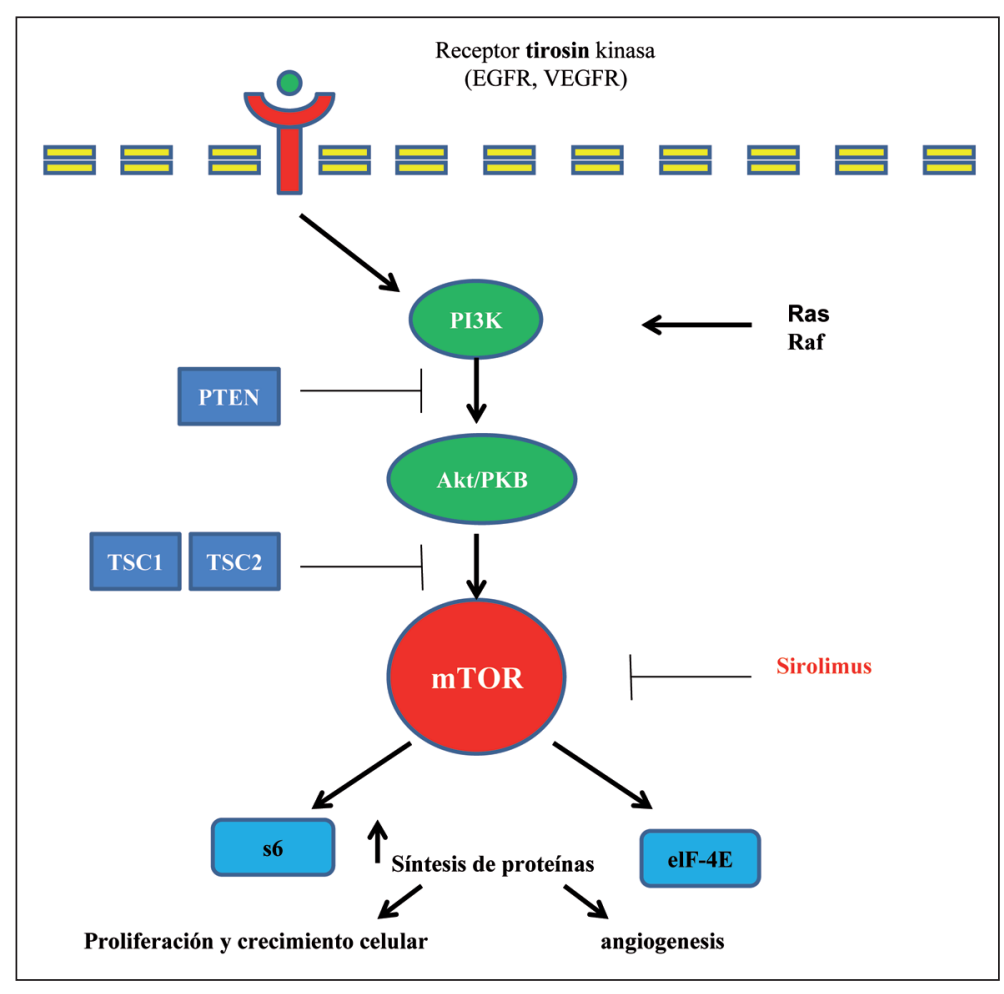

Figura 7. Vía PI3K/mTOR y mecanismo de acción de Sirolimus. Las señales de factor de crecimiento extracelular son transmitidas a través de receptores tirosin kinasa a PI3K. Las señales son transferidas posteriormente por medio de Akt/proteína kinasa B a mTOR que activa la síntesis de proteínas resultando en el aumento de la proliferación celular y la angiogénesis. Sirolimus inhibe directamente mTOR y previene la subsecuente proliferación celular y angiogénesis. mostraron mejoría en su estado clínico, con disminución del tamaño tumoral, normalización del recuento plaquetario y de los exámenes de coagulación, desapareciendo el riesgo vital e incluso logrando la remisión total de la lesión en un paciente. No observamos reacciones adversas manteniendo niveles en rango recomendado. Se desconoce si la suspensión del tratamiento, luego de 1 a 2 años, puede producir rebote del crecimiento tumoral, por lo que los pacientes deben mantenerse en control periódico.

\section{Conclusiones}

De acuerdo a esta experiencia en pacientes pediátricos con HEK, que es la primera publicada en Chile, el uso de SRL sería efectivo en el tratamiento de HEK refractario con FKM severo, permitiendo que ambos pacientes que presentaban graves complicaciones, sobrevivieran. No observamos efectos secundarios atribuibles a SRL, por lo que representa una opción terapéutica segura $\mathrm{y}$ disponible en nuestro medio hospitalario. A pesar del uso de SRL en otras patologías pediátricas como trasplantes de órganos sólidos, creemos que hasta no contar con más experiencia protocolizada en niños con HEK, no debería ser utilizada como terapia de primera línea, sino en casos en que fallan otras alternativas terapéuticas, por el riesgo de aparición de "segundos tumores"15.

\section{Referencias}

1.- Croteau S, Liang M, Kozakewich H, et al: Kaposiform Hemangioendothelioma: Atypical features and risks of Kasabach-Merritt Phenomenon in 107 referrals. J Pediatr 2013; 162: 142-7.

2.- Adams D, Wentzel MS: The Role of the Hematologist/ Oncologist in the Care of Patients with Vascular Anomalies. Pediatr Clin N Am 2008; 55: 339-55.

3.- Mukerji S, Osborn A, Roberts J, Valdez T: Kaposiform hemangioendothelioma (with kasabach merritt syndrome) of the head and neck: case report and review of the literature. International journal of pediatric otorhinolaryngology 2009; 73: 1474-6. 
4.- Hammill A, Wentzel MS, Adams D, et al: Sirolimus for the Treatment of Complicated Vascular Anomalies in Children. Pediatr Blood Cancer 2011; 57: 1018-24.

5.- Lyons LL, North PE, Mac-Moune Lai F, Stoler MH, Folpe $A L$, Weiss $S W$ : Kaposiform hemangioendothelioma: a study of 33 cases emphasizing its pathologic, immunophenotypic, and biologic uniqueness from juvenile hemangioma. Am J Surg Pathol 2004; 28 (5): 559-68.

6.- Fernández I, López J, Ramírez G, Márquez C: Vincristine -Ticlopidine- Aspirin: An effective therapy in children with Kasabach-Merritt phenomenon associated with vascular tumors. Pediatric Hematology and oncology 2010; 27: 641-5.

7.- Blatt J, Stavas J, Moats-Staats B, Woosley J, Morrell D: Treatment of Childhood Kaposiform Hemangioendothelioma With Sirolimus. Pediatric Blood Cancer 2010; 55 : 1396-8.

8.- Hauer J, Graubner U, Konstantopoulos N, Schmidt S, Pfluger T, Schmid I: Effective treatment of Kaposiform Hemangioendotheliomas associated with KasabachMerritt phenomenon using four-drug regimen. Pediatr Blood Cancer 2007; 49: 852-4.

9.- Chiu Y, Drolet B, Blei F, et al: Variable response to propanolol treatment of Kaposiform Hemagioendothelioma, Tufted Angioma, and Kasabach-Merritt phenomenon. Pediatr Blood Cancer 2012; 59: 934-8.

10.- López V, Marti N, Pereda C, et al: Successful Management of kaposiform hemangioendothelioma with Kasabach-Merritt Phenomenon using vincristine and ticlopidine. Pediatric Dermatology 2009; 26 (3): 365-6.

11.- Roque J, Ríos G, Hepp J, et al: Rescate con Sirolimus por rechazo agudo recurrente y diabetes mellitus en trasplante hepático. Caso clínico pediátrico. Rev Med Chile 2005; 133: 1221-4.

12.- Roque J, Ríos G, Vignolo P, et al: Sirolimus en trasplante de órgano sólido pediátrico. Experiencia en 5 casos. Rev Med Chile 2008; 136: 631-6.

13.- Adams D: Special considerations in vascular Anomalies: Hematologic Management. Clin Plastic Surg 2011; 38: 153-60.

14.- Hall GW: Kasabach Merritt Syndrome: pathogenesis and management. Br J Haematol 2001; 112 (4): 851-62.

15.- Drolet B, Trenor C, Brandao L, et al: ConsensusDerived Practice Standards Plan for Complicated Kaposiform Hemangioendothelioma. J Pediatr 2013; 163 (1): 285-91. 